\title{
7. Qualitative longitudinal research in family sociology
}

\author{
Laura Bernardi
}

\section{INTRODUCTION: WHEN QUALITATIVE RESEARCH GOES PROSPECTIVE}

The combination of qualitative interpretative approaches and longitudinal research designs, although not new in the social sciences, is gaining increasing recognition as a useful and even necessary avenue to explore family changes as they occur. Qualitative methods, whether longitudinal or not, emphasise the understanding of meanings, subjective experience, and agency. The use of such methods can improve our comprehension of how people choose a given alternative and reject another, and how they adjust or fail to adjust to their living circumstances. At the core of qualitative investigation is also an interest in understanding the meaning of the experiences of social actors in their social contexts. In family research, numerous one-off studies have investigated how different individuals and groups create, interpret, and negotiate family relationships, roles, and transitions. The longitudinal approach adds to this tradition by focusing on the course of events, as well as on the processes of change, stability, and continuity through time. At the individual level, the combination of qualitative and longitudinal research enables us to capture how individuals create meanings about the turns of their lives and their circumstances as they unfold. For instance, it is indispensable to investigate how processes like those initiated by a chronic disease, a union dissolution, or the experience of social discrimination unfold from specific socio-cultural and biographic perspectives. While one-off qualitative approaches are widely used in family sociology and have often collected retrospective information, until recently, longitudinal prospective methods were more popular in quantitative studies. ${ }^{1}$ They are generally used to describe and measure the objective aspects of change, such as personal and social trajectories, like family relations and biographic events associated with educational, employment, and residential careers. While quantification assesses the regularities and the probability of the occurrence of social dynamics, such data are thin when it comes to accounting for the logic of the actions that underlie them. In contrast, by investigating agency and meanings, qualitative longitudinal research (QLR) has the potential to unravel the complexity of changes that are involved in the making and the unmaking of family relationships across the life course. This will not come as a surprise if we think of both agency and meanings as dynamic objects of study: individual agency co-produces the processes through which individuals live and, therefore, their experiences of change. Subjective meanings are shaped by social interactions, and shift in relation to life course events and transitions.

QLR has started accumulating rich data on relational practices and multidimensional experiences of family and childhood across the life course, with change and temporality being central to their interpretation. Change can be conceived and qualitatively analysed at levels beyond just that of the individual, including at the couple, group, institutional, and societal 
levels. For instance, family researchers have looked at how couples live through the transition to parenthood before, during, and after the birth of the first child (Girardin et al. 2019; Grunow and Evertsson 2016; see also Grunow in this volume); or at the moment of divorce and during a given period after it (Smart and Neale 2007). At the group level, researchers have observed how the social networks of intimate ties are agentively mobilised and reshaped around a major family transition like a birth or a death (Bidart 2008; Hollstein 2002). At the institutional level, the focus may shift to changes in family laws (i.e., homosexual couples' rights to marry, adopt, or have a child; the regulation of rights and duties between ascendants and descendants) and family policies (i.e., parental leave or public child-care provision policies), and the ways these laws and policies are constructed, justified, and negotiated within a given society. In all cases, prospective qualitative research would aim to shed light on the subjective and processual character of social life.

As well as following individuals, groups, or institutions, QLR has the potential to capture the shifting temporal nature of the data, and, therefore, the researcher's temporal perspective. The temporal orientations of the data reflect the fact that they may be retrospective (narratives of life history), prospective (private letters), or both (repeated interviews). Similarly, at the moment of data analysis, the temporal gaze of both the participants and the researcher is often multidirectional, since present, past, and future may be anticipated and reinterpreted (Neale 2020; Sanchez-Mira and Bernardi 2020). Qualitative longitudinal studies often combine prospective follow-ups with retrospective biographic narratives of the past in their data collection (Scott and Alwin 1998). While longitudinal data can be purely retrospective, a prospective approach makes it possible to collect data that represent different 'presents', and to enrich the analyses with the present interpretation of the different 'pasts' and the different 'futures'.

QLR is, of course, not new, and it existed before it was labelled as such and identified as a separate, specific research approach by a number of recently published methodological handbooks. QLR has an established presence in several disciplines, including anthropology, criminology, psychology, social policy, and sociology. Consequently, a variety of disciplinary and sub-disciplinary terms, like prospective, follow-up, and qualitative panel studies, are often used interchangeably to refer to QLR (Holland et al. 2006). Examples of the most commonly used QLR designs include ethnographies of small communities over time, research conducted in the same fieldwork setting with long time intervals between visits, repeated interviews at given intervals of different durations, and cross-generational research.

These QLR approaches also have different time scales, with some designs being more time-intensive, and others being more time-extensive (Epstein 2002). Generational studies and community studies can stretch over decades (Brannen 2006). In the case of repeated interviews, participants are typically observed over at least two or more regular intervals of months or years. This latter approach is typically used in family research (see Section 2), but is also very frequently applied in youth research that looks at educational aspirations, choices, and future planning (Woodman 2011); and in health research that examines illness or recovery trajectories (Fadyl et al. 2016; SmithBattle 2018). Longitudinal video documentaries such as Seven Up, ${ }^{2}$ Child of Our Time, ${ }^{3}$ and Les bonnes conditions ${ }^{4}$ are popular and fascinating series of repeated filmed interviews that have followed youngsters of various social backgrounds across their life course transitions, from childhood, to adolescence, to well into adulthood.

Recently, a few methodological overviews have been published in the form of books or special issues that have pointed out the similarities among the different types of qualitative methods used in longitudinal research (Hermanowicz 2003; Neale 2020; Saldana 2003; 
Thomson and McLeod 2015). Such overviews were theoretically informed by the main principle of the life course paradigm (Elder et al. 2003), which states that the interdependencies between individual life trajectories, the societal conditions in which they evolve, and their unfolding through time are central to explaining both individual development and social processes (Bernardi et al. 2019). In this chapter, I first draw on these synthetic overviews to describe the essential features of QLR, which include change and temporalities. I then present a few studies to highlight the relevance of taking a longitudinal qualitative approach in family sociology. In Section 3, I outline some of the most important challenges that can arise in the conception and the realisation of QLR. Finally, I outline what appear to be the most promising emerging paths in QLR.

\section{SUBJECTIVITY AND TEMPORALITIES}

Most qualitative research engages with the concepts of human agency, meaning making, and subjectivity as the actor's personal interpretation of his/her own experience (Heinz 2016). This type of research is invaluable for generating new research questions and advancing sociological theory. In sociology, QLR has been mostly employed to look at how people decide upon or adjust to life course transitions. While agency, meaning, and subjectivity are central to all qualitative approaches, longitudinal qualitative research has a fundamentally different focus, as it can be retrospective, prospective, or both. The most important difference between longitudinal and other types of qualitative research is that in the former, time plays a role in the interpretation of the results. Most longitudinal family research is based on a retrospective biographic component that is integrated into a one-time study, which results in the collection of life histories (Riessman 1991). The focus of life histories is to capture the subjective sense-making and causality assumptions of individuals with regard to the decisions and the outcomes that shaped their biographies. A retrospective approach is longitudinal, since it reconstructs the past and individuals' accounts about life course changes; and it can also be projected into the future by capturing individuals' present reflections, aspirations, fears, and intentions.

It has been argued that one-time retrospective approaches suffer to a greater or lesser degree from the deterioration of reliability and validity concerning the past, particularly if the time scale is too long (e.g., older people's life histories). However, research in social psychology has indicated that such biases in personal life narratives are important components of the self (McAdams 2008), as life narratives represent 'internalized and evolving stories of the self that people construct to make sense of their lives in time' (McAdams 2005, p. 241). Such narrative identities (Singer 2004) reconstruct the past in a selective way and anticipate the personal future, infusing biographies with coherence and purpose as life goes by (McAdams 2008). Similarly, the literature on biographic narratives shows that with the accumulation of new experiences or changes in motivations or concerns, the meanings people associate with past events may also change, with some taking the spotlight and others fading out (Hareven and Masaoka 1988; Schütze 1980 cited in Bertaux and Kohli 1984, pp. 222-3). These ideas connect with Flaherty's $(1999,2003)$ notion of 'time work'. An individual's 'time work' is the efforts $\mathrm{s}$ /he makes to promote or supress particular forms of temporal experience by controlling or manipulating duration, frequency, sequence, timing, and allocation. 
One-time biographic narratives do not allow for comparisons of closer and more distant accounts of the same event or transition, and of the various perspectives that emerge during critical life course phases. By contrast, repeated interviews allow for both the collection of more recent accounts and the analysis of changes in subjective perspectives over time. A QLR approach has a specific interest in the continuously evolving interpretation of the past and the future under subsequent present conditions.

However, not all qualitative research over time is longitudinal. In addition to being based on a design that includes data collection over time, QLR has theoretical and analytical frames that explicitly address processes and temporality; i.e., that change over time together with the mechanisms of its maturation (Saldana 2003). As Thomson et al. (2003, p. 185) observed, what distinguishes longitudinal qualitative research is 'the deliberate way in which temporality is designed into the research process making change a central focus of analytical attention'. In other words, QLR is performed not just over time, but through time. Thus, QLR explores the nature of time, or the temporal dimension of experiences.

QLR focuses on the understanding of temporal processes by means of a processual analysis (Abbott 2016). Processual analysis considers processes in a narrative perspective, whereby the weight of each of the different elements of the process depends less on its presumed objective relevance, and more on the extent to which it makes sense to the subjects, and on how this sense shapes their future orientations. By focusing on the prospective character of longitudinal research, we improve our comprehension of the relationship between present situations and narratives, future actions, and retrospective meaning making. For instance, in biographical interviews with elderly individuals (over age 75), Bornat and Bytheway (2010) clearly showed that time modified the individuals' interpretations of their experiences by comparing the interview material with the individuals' personal diaries at younger ages. Thus, the retrospective views of past experiences provided at older ages contrasted with the emotions and contingencies of the present that were captured in the diaries. Here, the prospective component is composed of the written data of the diaries, which were written following or anticipating the vagaries of people's lives.

To better clarify how temporality is central to qualitative longitudinal analyses, I will extract an illustrative case from the ongoing project 'The multiple paths of lone parenthood' (Bernardi and Larenza 2018). The data collected include narrative interviews, biographical calendars, and social network data. The longitudinal research design of the project was to generate retrospective biographical data that reconstruct the transition to lone parenthood within a prospective research design component in which objective and subjective changes were tracked. The case study is that of Anouk, a lone mother in her 30s. During the three interviews conducted over six years, Anouk reported on her transition to lone parenthood, and on her troubled relationship with the father of her child in the period that followed. At wave 1, she had left the common household to go back to live with her parents, after a back-and-forth period in which she had got her hopes up on several occasions that her partner would be sober enough to visit their child, who was aged four at that time. While the event that led to Anouk moving out of the couple's home was that he once again drank during his medical treatment, the decision to leave was clearly also based on accumulated stressors that were interpreted retrospectively as a series of 'trigger points' that eventually reached a point of no return. Two years later, at wave 2, Anouk had resumed an ambivalent relationship with the father of her child. The father said he would take care of the child more frequently, and he even slept in their apartment occasionally. At this point, Anouk's aspirations for the future were to become 
a 'regular' family again. After three more years, at wave 3, the couple had separated again, and this time definitively, according to Anouk. At this point, her emotional descriptions of the ups-and-downs of his drinking trajectory and her hopes and feelings of betrayal had been substituted by a coherent reconstruction of a relationship that was doomed to fail from the start. Having definitively separated from her partner, Anouk was framing these previous events and circumstances within a lifetime horizon in which the contingencies of everyday life were accompanied by emotional and temporal distance.

This case shows how events, motivations, and emotions in the past are reinterpreted in the present in order to give sense and coherence to biographies in the making. QLR approaches provide unique empirical data on family processes as they are experienced. On the one hand, the ability to observe and situate individuals' changing perspectives over life course transitions across different present times (at each subsequent wave of data collection) distinguishes prospective QLR from both one-off qualitative analyses and life histories. On the other hand, QLR complicates the understanding of processes over time that are typically investigated by quantitative longitudinal analyses, because it adds to the information on the durations and the sequencing of events and transitions, the subjective meanings that are progressively attributed to life trajectories in the making, and the related experiences of time throughout these processes.

\section{EXAMPLES OF PROSPECTIVE QUALITATIVE RESEARCH IN FAMILY RESEARCH}

There has been an increasing interest in family research in the temporal unfolding of lives, and of notions like 'trajectories' or 'careers' (e.g., partnership trajectories). In family research, quantitative longitudinal research is now commonly used to examine life trajectories, events, and transitions, as well as changes in attitudes and values related to family practices and regulations. Sophisticated statistical methods of description and analysis, such as sequence and event history analysis, have flourished, making it possible to test hypotheses regarding the determinants and the consequences of family dynamics. However, while such methods are tailored to test hypotheses, they are less useful for generating new hypotheses. Developments in family theory often come from analyses of people's subjective experiences, which consist of the meanings and the expectations associated with family events, transitions, and relations; and of their changes over time and through time (e.g., how people respond to different forms of relationship losses and family reconfigurations).

While much of this prospective research is quantitative, the few existing prospective qualitative studies that have been conducted have revealed the subjective side of family trajectories. Most of these studies were based on repeated interviews of research participants who were selected to report on a particular experience, event, or transition (e.g., divorce, parenthood, widowhood), and were then followed over more or less regular time intervals. In other prospective qualitative studies, the research participants were chosen because they belonged to the same age group, cohort, or community. In this section, we examine the added value of QLR for family research. Providing a complete overview of the range of approaches available in prospective qualitative research exceeds the scope of this chapter, which concentrates on the method used most frequently in family research: namely, conducting repeated waves of interviews. ${ }^{5}$ Studies of children and youth in particular have employed repeated biographical inter- 


\section{Research handbook on the sociology of the family}

views with participants to follow them across their differentiating lives and transitions. While these kinds of studies are usually independent and self-contained, they are sometimes - albeit far too rarely - related to larger population-representative panels (Legewie and Tucci 2020).

\subsection{Experiencing Family Processes}

The more important contribution of prospective qualitative research to family studies is that it allows researchers to study family processes as they are being lived through by the actors. Thus, this research approach is able to capture meanings, norms, hopes, and fears around key life events and transitions, such as marriage, birth, parenting and grand-parenting, divorce, and the death of intimates; as well as related processes, such as the formation and dissolution of relationships, or negotiations between parents about home and work responsibilities.

In their study of youth transitions in France, Bidart and Lavenu (2005) followed young men and women aged 17 to 23 over three years. In each of the three waves, the development of the young people's personal networks was linked to the events marking their entry into adult life. The results showed that partnership formation affected the young adults' sociability by first expanding the size of their social networks, because partners pool friends; and, then, when the new couple had moved in together, by reducing it, as domestic life was prioritised. The study also found that having a child further contributed to couples' 'isolation'. While similar trends have often been reported in quantitative analyses of social networks and family formation, the prospective qualitative approach shows that the restriction in sociability that came with forming a union had positive connotations for young couples and parents; i.e., that they seemed to appreciate the out-selection of superficial relationships, and the calmer and less scattered lifestyle that followed (Bidart and Lavenu 2005).

Keddi et al. (2003) studied the changing work and family life plans of women in eastern Germany around the time of German reunification, and in the period thereafter. Over seven years (1991-98), they conducted four interviews with an initial sample of 125 women who were born in Saxony and Bavaria, and were aged 18-27 at the time of the first interview. The researchers were interested in understanding how the women were thinking about their future, and followed them while their biographies were unfolding starting in early adulthood. The results showed that the differences in the choices and the scopes of the women's lifestyles were shaped not only by their life circumstances, but also by 'life themes'; and that the latter were already emerging in the women's early adolescent years, and were often maintained as life orientations thereafter. The study found that the eastern German women of these cohorts uniformly described work and family as parallel and dominant life themes, whereas the western German women reported multiple life themes.

Girardin et al. (2019) interviewed 31 Swiss couples three times: during pregnancy, four to six months after childbirth, and about a year after childbirth. Using qualitative materials drawn from these three waves, they collected data during pregnancy, at a point when parenthood had become an everyday reality, and at a point when the mother had returned to her job after maternity leave. They analysed the intention to share tasks after parenthood and the reasons for succeeding or failing to do so. Couples who had planned to have a non-normative (more gender-egalitarian) division of tasks after parenthood did not always manage to do so. The researchers showed that there was variation in the extent to which men and women adapted to their new care obligations, but also that there was less variation in the arguments they gave for doing so. This was partly because they were not able or willing to resist cultural and institu- 
tional settings that pushed them towards adopting more traditional practices and roles during the transition to parenthood. At the couple level, economic and career considerations were frequently mixed with the internalised norm that the mother was the natural primary caregiver, even in the rare cases in which the woman was earning more than the man. The study also found that Swiss family policies that put less pressure on couples who adopted a traditional one-and-a-half earner model, while penalising couples who adopted a dual-earner model, played a key role in the couples' decisions. Only by capturing the fundamental coherence of both partners' perspectives through subsequent open questions about the meanings and the subjective experiences associated with the adjustment process were the researchers able to capture the interdependence of individuals' agency and the context of shared meanings.

In the examples above, researchers conducted repeated interviews with the same individuals at different moments in time. This approach enabled them to compile separate datasets for each survey wave that represent different real 'presents' in which the individual and the contexts are synchronously discussed in their objective and subjective aspects. The collection of data on the individuals' evolving subjective perspectives, as well as on objective biographical developments, enables researchers to better understand family transition processes, and to contextualise them within subsequent historical periods. Thus, it is possible to compare people's situations and life plans with their achievements, but also with their plans at another point in time (Longo et al. 2013). Changes in values can be examined, including the effects of background social factors on values, particularly changes that occur in response to lived experiences, such as work experiences (Johnson 2002).

\subsection{The Relationship between Institutions and Families}

Longitudinal prospective designs allow researchers to study how biographies intersect with policies and practices. How such an unfolding process can shape lives through priority setting in changing circumstances was explored by Millar and Ridge's (2013) 'Family Work Project'. This project is a qualitative prospective study of the everyday management of work and care responsibilities by low-income British lone mothers enrolled in a welfare-to-work programme, which was designed to facilitate the transition from being a social benefit recipient to being a working mother. The study examined the views of 50 mothers and 61 children (aged eight to 14 at the time of the first interview) between 2004 and 2007 (three waves of interviews). The discussion of the findings highlighted that the sustainability of paid employment was constantly questioned by the participants, because being a working lone mother meant involving the whole family in a different configuration, and was not just about one person having a job. Understanding what forms of agency a mother and her children had when taking a job, arranging care, and claiming tax credits and benefits implies understanding the margins of choice they had, and how the policies worked in practice. The mothers were continuously exploring opportunities for adjustments over time, since changes in employment meant that they had to find new job-compatible arrangements. In reporting the many challenges they faced, these mothers made clear that their aim was to integrate their employment into everyday family life and practice, and that they would evaluate any solution or compromise based on whether they thought it was in the best interests of their family. Thus, in their view, work had to fit with family life, and not vice versa. As this example shows, understanding family functioning and configurations in their social context is important not only to advance social theory at a time when families and family relationships are becoming increasingly diverse, but to improve our 


\section{Research handbook on the sociology of the family}

ability to define the policy priorities affecting families, and the efficacy of such measures. A qualitative approach can tell us how a policy programme is being delivered, the range of issues that emerge among programme participants, and how the programme is being received. QLR is suitable for evaluating family policies, as it can increase our understanding of the delivery, impact, and durability of outcomes from interventions.

A study of the transition to lone parenthood in Switzerland based on interviews with 40 lone parents who were interviewed shortly after the transition to lone parenthood and three years later highlighted the moral dilemmas mothers sometimes faced when they tried to claim their rights in relation to their children's non-residential parent (Larenza 2019). For instance, the analysis showed that some of the mothers who did not receive child maintenance payments from their former partner did not act in response to this violation of their rights. The main reason they gave for failing to do so was that they believed that starting a legal procedure and filing a claim could endanger their children's relationship with the other parent. Some mothers tried to adjust to such situations by increasing their own income from paid work, which had repercussions in other life domains. For example, when these mothers increased their working hours, they often struggled to find appropriate childcare (Struffolino et al. 2020). The author concluded that even welfare institutions that are designed to protect lone parents against poverty and overburden fail to realise that lone mothers' choices are primarily guided by their 'ethic of care'. Post-divorce mothers showed a similar level of reticence in negotiating divorce terms in the study conducted by Smart and Neale (2007). The study analysed the shifting family practices at the moment of divorce, and 12 months later. During this transition period, in which the former partners were negotiating care arrangements and maintenance duties, moral dilemmas often arose. They found that in some cases, the mothers were not able to end what had been an unequal relationship through separation or divorce when co-parenting was involved; and that they often accepted unequal arrangements, as their choices were morally motivated (avoiding harm to children and sustaining connections with the father), even when these choices were very costly for themselves.

These studies reveal that when parents negotiate union break-ups and their consequences, they consider the available options in relation to significant others, even when conflict is present. For example, mothers are often willing to take into account their ex-partner's financial hardship or health difficulties during negotiations. Such findings have important consequences for family law professionals and for social policies, since they illustrate that family members remain related through moral dilemmas constructed around the perception of other people's needs that go beyond the legal and policy rationales. Including what matters to people is crucial to ensure that interventions work and that laws are perceived as fair.

\subsection{Populations Who Are Hard to Reach or Hard to Follow}

The third added value of prospective qualitative studies in family research is that they can address populations who are hard to interview in a survey, such as underage children or women who are victims of abuse and violence (Burton et al. 2015); or populations who are hard to study through larger panel data collection efforts because their initial sample sizes are small, and because the likelihood of losing them to attrition over time is relatively high, such as the fathers of children born to underage mothers or lone mothers on social assistance benefits.

Neale and Flowerdew (2003) showed that children who were interviewed at the time of their parents' divorce, and then four years after the divorce, adjusted to subsequent family 
transitions in an active and often resilient way. The study found that the pace and the nature of changes in the children's families, but also in other areas of their lives, defined the concerns they had between ages 11 and 17 , and their long-term reflections on post-divorce family life. The longitudinal aspect of this research could be used to explore whether the children's views and feelings were in line with presumptions made by the legal and welfare systems about their best interests through the various post-divorce phases (Smart et al. 2001). When one of their parents re-partnered, the children said they appreciated the economic benefits and the new activities they were able to engage in with the stepparent, as well as their parent's increased happiness. At the same time, they were critical about having to negotiate new rules and to adapt to stepsibling relationships. Thus, it appears that children's 'psychological travelling time' (Giddens 1992, p. 103) from one situation to the next is crucial for understanding the process leading to children's outcomes after parental separation. Public policies designed to support children in overcoming parental break-ups should therefore consider children's experiences in such circumstances.

Neale and Patrick (2016) followed 12 low-income young fathers, and described the shifting nature and the quality of their relationship with the mother of their child. The longitudinal aspect focused on the fathers' engagement with their child over five waves of interviews between 2010 and 2014, and a refreshment sample over 2013-14. These men learned they were going to be a father at a young age (half of them before age 16, the rest before age 24); some were in a relationship with the mother at the beginning of the study, while others were not. The interdependent nature of the sexual, relational, familial, socio-economic, and health-related issues that arose in the transition to fatherhood, and in the subsequent relationship with the child, were explored through the repeated, open-ended interviews. The analysis identified the main factors that explained the different degrees and kinds of engagement these fathers displayed in their relationship with their child. The study concluded that 'the ethos of engaged fatherhood' (Neale and Patrick 2016, p. 33) required elements that were often not necessarily within reach for these young men, such as material and emotional resources for joint parenting; adequate standards of care; emotional maturity and commitment; and a positive relationship with the mother of the child. Based on this perspective, policies designed to improve the situations of children born to underage parents could be adjusted to empower young fathers to engage in a sustained relationship with their new-born child by helping them build their relational skills.

From the few examples above, it appears that QLR is already enhancing the knowledge we have on family configurations and dynamics that comes from large-scale panel studies. It contributes to family research by zooming in on the subjective experiences of events and transitions, which can improve our understanding of how biographical pathways within families are constituted and negotiated, and what ethical considerations and life themes are guiding people in these situations. Thus, QLR can shed light on moral dilemmas in which the actor must consider two or more moral values, but can only honour one of them. Such situations are abundant, especially during family transitions that create critical disruptions of the previous normative order. QLR can be used to document changes and adaptations in family configurations that result from non-normative trajectories by stressing the significance of the timing and timeliness of these transitions, and the perspectives of actors who tend to be less heard in such processes, like minor children. 


\section{CHALLENGES AND OPPORTUNITIES IN QUALITATIVE LONGITUDINAL RESEARCH}

Despite its unique strengths for understanding family dynamics in the ways discussed in the previous sections, QLR is still less developed in family sociology than it is in qualitative cross-section studies or quantitative panels. This is in part because the opportunities opened up by QLR are counterbalanced by its substantive challenges. While some of the challenges that arise are common to all longitudinal research (high costs, time scales, number of passages and their frequency, sample attrition and retention), others are specific to QLR, particularly problems related to managing flexibility in research design and analysis, multiple analytical perspectives on time and change, and the ethical questions raised by having repeated and often highly personal research interactions with participants.

\subsection{Flexibility}

A major advantage of QLR - which also represents one of its major challenges - is its open character and flexibility. On the one hand, development and innovation can take place throughout the entire research process. On the other hand, changing samples, protocols, and the analytical focus requires that researchers are able to describe in fine detail all of the sources of variation that accumulate over time (Koro-Ljungberg and Bussing 2013; Vogl and Zartler 2021).

Among the decisions involved in the process of data generation are decisions about the number and the frequency of data collection episodes, which primarily depend on the phenomenon under study (Saldana 2003). These aspects are not always anticipated. For instance, a one-time visit can span several decades through generational or family chain sampling, as in the case of the study 'The Making of Modern Motherhood' (Thomson 2011), which analysed the critical moment of biographical and family change, the transition to parenthood, as the arrival of a new generation in the extended family. Six of 12 intergenerational chains were followed up two more times, which created an intergenerational sample that allowed researchers to study in depth the process of changing configurations in family relationships after parenthood. In such cases, the separation between the research design and the research process decreases. Since a prospective longitudinal approach requires researchers to adapt to what they learned in the previous wave in order to address change in the subsequent wave, making decisions about data generation over time becomes an iterative and complex process. Accordingly, the study can grow by, for example, progressively including younger or older generations; or it can contract over time.

The flexibility of QLR may affect sampling. Progressive sample focusing carefully selects sub-samples that can be singled out for more detailed attention or more intensive tracking, and leaves aside the rest of the available baseline sample (Pollard 2007). The chosen sub-samples are likely to offer greater insights into the themes of the study by, for example, exemplifying those individuals undergoing further transitions, or those individuals with particularly distinctive or diverging trajectories. The full baseline sample may then be followed up with less intensity and/or frequency (e.g., interviewing via telephone or online rather than in person). Shirani et al. (2012) drew on two case studies of men who became fathers for the first time in 2000 from a wider sample of 46 men. The funnelled second-wave sample focused on the 
men's relationship to involvement and the linked concepts of exclusion and redundancy; i.e., on terms that emerged from the data and changed over time in the men's accounts.

Flexibility can also characterise fieldwork timing. Understanding family change over time can involve mapping the related changes in circumstances. Since it is hard to say when change will occur, it is a strength of the prospective approaches that it can be adapted to the timing of the fieldwork. In a study of people undergoing a degenerative disease, the researchers decided to have monthly telephone calls with half of their sample in addition to having biannual face-to-face interviews in order to compensate for the high attrition due to death (Carduff et al. 2015). This level of contact intensity allowed the researchers to gain a real-time impression of how the illness progressed, and to map the ups and downs typical of people facing cancer. Extending such a flexible approach to delicate and dynamic processes in family research such as domestic violence, child custody arrangements, and parent-child relationships during major transitions, like leaving home - would be straightforward, and would likely be a fruitful research endeavour.

Flexibility in QLR means not only that the sample and the timing can be modified, but also that the researchers and the respondents face additional challenges. Compared with longitudinal retrospective qualitative approaches, QLR is costlier not only in terms of the budget, but also in terms of the amounts of time and the levels of engagement and flexibility required of the research team and the participants. Specific extra measures are needed to attach participants and researchers to the project (Farrall et al. 2016; Weller 2010). Moreover, efforts to retain the sample are needed for smaller samples, as attrition may be a crucial element in the saturation criteria; and for larger samples, as rates of researcher turnover may be higher, which can make sustaining the trust of participants more difficult (Dwyer and Patrick 2020).

Finally, flexibility also applies to the protocols and the mode of data collection over time. In the wake of developments in online panel surveys, qualitative research has also turned to online interviewing (Salmons 2015). While this approach has advantages (interviews can be arranged regardless of the participants' location and other scheduling constraints, which can reduce attrition and costs), researchers have warned that it puts at risk the levels of involvement, flow, intimacy, and intensity of the exchanges and the rapport. Specifically, for qualitative research, in which trust between the researchers and the participants is needed to ensure data quality, and non-verbal communication is an important component of the relationship, these risks cannot be overlooked. As well as representing a psychological barrier, using technology implies that technical preparation is needed from both sides to ensure that no problems that could interrupt the communication flow occur. While such limitations are certainly relevant for one-shot studies or for first-wave panels, they are only partially limiting in an ongoing study. If a first contact has already been established and a commitment does not have to be built, web interviews can help to consolidate the research relationship.

\subsection{Multiple Perspectives on Time and Change}

In prospective qualitative research, the boundaries of the study design and the participants' expressions are less 'closed', and time offers a 'continuously changing point of reference for their analysis' (Blossfeld 1996, p. 182). The analysis of temporal data combined with an interpretative approach may be conducted, very generally speaking, in two ways. Research could be conducted iteratively over each point in time for which data are collected, or it could be conducted using a summative approach that considers all waves together once the study 
is over. These modes are not mutually exclusive, but they assume different perspectives on the temporal frames considered in the analyses. Process and change remain central to both procedures, but the iterative approach emphasises variations among points in time, while the summative approach looks at what is produced by their cumulative or blended effect.

In three studies that examined various aspects of the lives of Polish immigrants in the United Kingdom, Ryan et al. (2016) re-interviewed the respondents over intervals of several years, even though there had not been a longitudinal design in the original plan. Having adopted an iterative approach, the researchers reported that after the follow-up interviews, they had to challenge their previous interpretation of the data, as the contingency of their previous accounts and interpretations became clear. McKie et al.'s $(2001,2002)$ analysis of gender, caring, and work showed that employed mothers manage work and care by living in multiple time horizons simultaneously. They labelled these time horizons 'caringscapes' (McKie et al. 2002, p. 908), thereby paraphrasing Adam's (2000) 'timescapes'. The mothers in their study continuously anticipated contingencies such as child illness, and daily scheduling challenges such as different patterns of school and childcare for each child, while also planning for the long-term changes in the needs of their family over the subsequent months and years. These different types of time horizons provide a framework for the analysis of longitudinal qualitative data that consider temporalities and time.

The extensive volume of data that QLR can capture, as well as the complexity inherent in such data, are disincentives to engaging in larger sample studies that could quickly become difficult to manage and analyse. The challenge is to find a way to scale up from small-scale qualitative enquiries while ensuring that economies of scale are realised, but also that the qualities of small-scale research are not lost.

\subsection{Scaling Up}

A direct approach QLR scholars have recently used to increase the return of small studies is promoting cross-fertilisation among existing projects. For instance, researchers can bring together several in-depth, small-scale studies to increase the generalisability and the cross-context reliability of the results. One pioneering example of such a collaboration in family sociology is the 'Timescape project', which combines existing data drawn from seven longitudinal datasets to facilitate their collective reuse (Irwin et al. 2012; Irwin and Winterton 2014). However, when teaming up by combining smaller studies, researchers need to have a reliable and standard procedure for archiving data, and a culture of using secondary data for qualitative analyses (Heaton 2008). Qualitative data archives have been actively promoted, particularly in the United Kingdom, since the 1990s, with the aim of encouraging the re-analysis of secondary data (Hughes and Tarrant 2020). Important qualitative research archives exist internationally that are intended to encourage methodological and ethical debates on data reuse, to reduce the underexploitation of collected data, and to encourage comparative research across time and space (Corti 2019). Therefore, secondary data analysis is increasingly possible, and data are increasingly accessible. However, because anonymity is difficult to maintain with rich biographic materials (Thomson 2007) that are updated with follow-up information from subsequent waves, there has been some resistance to such analysis in parts of the research community. In addition, some researchers have argued that not 'being there' makes it hard to interpret the data, and that the context of data production may be under- 
estimated. However, this position is not universal, and the secondary use of data is gaining ground in the QLR community (Irwin et al. 2012).

A second and more indirect way to scale up QLR studies is to anchor them in larger population-representative samples, and to combine breadth and depth through a data analysis. In biographical mixed-methods approaches, researchers pick theoretically selected cases belonging to a larger cohort study to compare them and to construct multicase narratives with the aim of better understanding how social structure and individual agency articulate in biographies. A notable example of the use of such an approach from the 1990s in family sociology is the research on the trends in family obligations and responsibilities by Finch and Mason (1993). The study matched representative patterns of kin exchanges with in-depth reflections on their meaning for respondents. A second example is the longitudinal mixed-methods study by Heinz et al. (1998) of young adults in two German cities with different job markets and unemployment rates in the 1990s. Combining the analysis of in-depth interviews (initially 120, and followed over 10 years) and panel data from which the interviews were drawn, they examined the link between the work and family biographies of young adults with vocational education and their planning of career and family transitions. The transnational Child Raising Arrangements project adds a layer of complexity, as it pursued a mixed-methods longitudinal study with matched samples of parents, caregivers, and children across nations. The aim was to situate outcomes concerning education, job performance, health, and emotional well-being in a cultural understanding of the family, of school functioning, and of migration law (Mazzucato 2015).

In mixed-methods approaches, the difference between the case and the variable generalisations over time can lead constructively to a sequential integration of standardised and narrative procedures, and create a dialogue that enriches our understanding of how structure and individual biographical management are linked. Trajectories such as life events sequences and the set of constraints in which they evolve can be described through the statistical analyses of large survey data, while biographical actions and their logic are illustrated by qualitative analyses. There is an increasing awareness of the complementarities between quantitative and qualitative components in longitudinal studies. However, there is still the danger that narrative and constructivist turns in the social sciences on the one hand, and the growing sophistication of holistic and structural equation modelling approaches on the other, will create a sterile divide in empirical studies in family sociology, thus reducing the opportunities for productive collaborative research.

\subsection{Ethical Concerns}

The development of QLR has been accompanied by a growing debate over the specific ethical problems connected with the high degree of proximity between the researchers and the study participants in repeated interactions. Long-term participation means that at each wave, whether a participant enters, leaves, or re-enters the field must be negotiated. Among the key issues that can arise are consent, intrusion, and study boundaries.

In QLR, consent must be given not just once, but should be a repeated process that starts at the beginning of the study, particularly when dealing with young people whose life perspectives and conditions can evolve rapidly over a short period (Saldana 2003). If participants decide to withdraw, special consideration should be given to whether and, if so, with what limitations the material from previous waves can be used. Confidentiality and anonymity 
also acquire more relevance when thinking about the potential impact of the research on participants over time, including the dangers of intrusion, dependency, and the distortion of life experiences through repeated interventions (Thomson et al. 2003). Ethical considerations concerning the researchers involved also increase in importance, as the risk of becoming overly emotionally involved with the fieldwork grows over time (Yates and McLeod 1996). Moreover, the boundaries of when and how to close a study have to be negotiated, particularly when researching children and young people or otherwise potentially vulnerable groups (Crivello 2017; Farrall 2006; Thurman 2015). With repeated interaction opportunities, the need for researchers to offer disclosure and assistance to the participants is likely to increase.

\section{CONCLUSIONS}

In this chapter, I argued for the complementary and unique value of adopting qualitative longitudinal methods in family sociology research. I limited myself to prospective studies, as this research design is still relatively small. In particular, I highlighted the importance of capturing the subjective perspective of family transitions and relationships, and of considering different temporalities when considering individual agency in family processes. I illustrated this point by presenting a number of QLR studies around family transitions, and showing how they contribute to the literature by exploring at least three areas that are made less visible through other methods: the logics of actions and temporalities underlying family trajectories and related choices; the complex relationship between individuals' priorities and family-related institutions; and the perspectives of hard-to-study populations. Finally, I noted some of the major challenges researchers face in engaging with QLR, such as the very flexible approach to data collection and to the interpretation of results, which also need to account for the multiple and sometimes contradictory perspectives on change. I briefly discussed the possibilities opened up by the establishment of qualitative data archives to do comparative research, and by mixed-methods panel studies to merge the breadth and depth of data collection and analysis. I concluded by noting the specific ethical concerns raised by QLR, such as those related to the repeated and emotionally close interactions between respondents and researchers, as well as the flexible character of their reciprocal engagement. Despite such concerns, QLR is an exciting methodological field that is currently being fully developed, especially as it offers opportunities for digital panels and participatory archive-based research (e.g., Thomson et al. 2018). Family sociology is faced with the challenge of studying increasingly dynamic biographies that result in complex interrelationships among life domains, among related individuals, and within fragmented institutional framings. Understanding the ways in which people experience and interpret their changing life circumstances contributes to our understanding of families as they are being made.

\section{ACKNOWLEDGEMENTS}

This publication has benefited from the support of the Swiss National Centre of Competence in Research LIVES - Overcoming Vulnerability: Life Course Perspectives, which is financed by the Swiss National Science Foundation (grant number: 51NF40-160590). 


\section{NOTES}

1. Both retrospective and prospective studies can be called longitudinal, as the researcher can reconstruct changes over time. However, retrospective studies are based on reports collected at a given point in time, while prospective studies collect data at subsequent time intervals, thus following change instead of reconstructing it. In the rest of the chapter, I use qualitative longitudinal research to indicate primarily prospective studies.

2. Paul Almond and, subsequently, Michael Apted followed up 14 seven-year-old children of various socio-economic backgrounds in Britain starting in 1964, with the explicit assumption that each child's social class would predetermine his/her future. Almost all have been followed up until their 60s in 2019 (Pearson 2012).

3. From 2000 to 2017 , Robert Winston followed the lives of 25 children born at the beginning of the twenty-first century, as they grew from infancy, through childhood and on to becoming young adults (BBC 1 2020). A similar documentary series by Anna Stickland, 'Born to be different', did the same with disabled children (Wyatt 2016).

4. From 2003 to 2016, Julie Gavras followed the transition to adulthood of eight adolescents from the high residential areas in Paris (Rousseau 2018).

5. Other methods consist of analysis of diaries, participant observations, and visual-based research; particularly in children, older people, and community studies (Neale 2020).

\section{REFERENCES}

Abbott, A. (2016), Processual Sociology, Chicago, IL: University of Chicago Press.

Adam, B. (2000), 'The temporal gaze: The challenge for social theory in the context for GM food', British Journal of Sociology, 51 (1), 125-42.

BBC 1 (2020), Child of Our Time, accessed 3 July 2020 at www.bbc.co.uk/programmes/b0072bk8/ episodes/guide.

Bernardi, L. and O. Larenza (2018), 'Variety of transition into lone parenthood', in L. Bernardi and D. Mortelmans (eds), Lone Parenthood in the Life Course, Cham: Springer, pp. 93-108.

Bernardi, L., J. Huinink, and R.J. Settersten (2019), 'The life course cube: A tool for studying lives', Advances in Life Course Research, 41, 1-13. doi:10.1016/j.alcr.2018.11.004.

Bertaux, D. and M. Kohli (1984), 'The life story approach: A continental view', Annual Review of Sociology, 10, 215-37. doi:10.1146/annurev.so.10.080184.001243.

Bidart, C. (2008), 'Dynamiques des réseaux personnels et processus de socialisation: évolutions et influences des entourages lors des transitions vers la vie adulte', Revue Française de Sociologie, 49 (3), 559-83. halshs-00325961.

Bidart, C. and D. Lavenu (2005), 'Evolutions of personal networks and life events', Social Networks, 27 (4), 359-76. doi:10.1016/j.socnet.2004.11.003.

Blossfeld H.-P. (1996), 'Macro-sociology, rational choice theory and time a theoretical perspective on the empirical analysis of social processes', European Sociological Review, 12 (2), 181-206. https:// doi.org/10.1093/oxfordjournals.esr.a018185.

Bornat, J. and B. Bytheway (2010), 'Late life reflections on the downturn: Perspectives from "the oldest generation"', Twenty-First Century Society, 5 (2), 183-92.

Brannen, J. (2006), 'Cultures of intergenerational transmission in four-generation families', The Sociological Review, 54 (1), 133-54. doi:10.1111/j.1467-954X.2006.00605.x.

Burton, L.M., D. Purvin, and R. Garrett-Peters (2015), 'Longitudinal ethnography: Uncovering domestic abuse in low-income women's lives', in J. Hall (ed.), Female Students and Cultures of Violence in Cities, New York: Routledge, pp. 37-88.

Carduff, E., S.A. Murray, and M. Kendall (2015), 'Methodological developments in qualitative longitudinal research: The advantages and challenges of regular telephone contact with participants in a qualitative longitudinal interview study', BMC Research Notes, 8, 142. doi:10.1186/s13104-015-1107-y.

Corti, L. (2019), 'Archiving qualitative data', in P. Atkinson, S. Delamont, A. Cernat, J.W. Sakshaug, and R.A. Williams (eds), Sage Research Methods Foundations. doi:10.4135/9781526421036813114. 
Crivello, G. (2017), 'Qualitative longitudinal research with children and young people', in T. Skelton, Ruth Evans, and L. Holt (eds), Methodological Approaches: Geographies of Children and Young People, Vol. 2, Singapore: Springer.

Dwyer, P. and R. Patrick (2020), 'Balancing sample breadth and qualitative depth: Exploring experiences of welfare conditionality in two prospective qualitative longitudinal studies', Longitudinal and Life Course Studies Journal. doi:10.1332/175795920X15913557982929.

Epstein, T. (2002), 'Mysore villages revisited', in R.V. Kemper and A. Royce (eds), Chronicling Cultures: Long-Term Field Research in Anthropology, Walnut Creek, CA: AltaMira, pp. 59-80.

Elder, G.J., M. Kirkpatrick-Johnson, Jr., and R. Crosnoe (2003), 'The emergence and development of life course theory', in J.T. Mortimer and M.J. Shanahan (eds), Handbook of the Life Course, New York: Plenum, pp. 3-19.

Fadyl J, A. Channon, A. Theadom, and K.M. McPherson (2016), 'Optimising 3 qualitative longitudinal analysis: Insights from a study of traumatic brain recovery and adaptation', Nursing Inquiry, 24 (2), e12170. doi:10.1111/nin.12170.

Farrall, S. (2006), 'What is qualitative longitudinal research', Papers in Social Research Methods Qualitative Series, 11, 1-25.

Farrall, S., B. Hunter, G. Sharpe, and A. Calverley (2016), 'What "works" when retracing sample members in a qualitative longitudinal study?', International Journal of Social Research Methodology, 19 (3), 287-300. doi:10.1080/13645579.2014.993839.

Finch, J. and J. Mason (1993), Negotiating Family Responsibilities, London: Routledge.

Flaherty, M.G. (1999), A Watched Pot: How We Experience Time, New York: New York University Press.

Flaherty, M.G. (2003), 'Time work: Customizing temporal experience', Social Psychology Quarterly, $66(1), 17-33$.

Giddens, A. (1992), The Transformation of Intimacy: Sexuality, Love and Eroticism in Modern Societies, Cambridge: Polity Press/Basil Blackwell.

Girardin N., D. Hanappi, and J.-M. Le Goff (2019), 'Couples’ alignment of pre-birth plans and post birth realities in Switzerland: Non-normative adaptation to the one-and-half earner model', in D. Grunow and M. Evertsson (eds), New Parents in Europe: Work-Care Practices, Gender Norms and Family Policies, Cheltenham, UK and Northampton, MA, USA: Edward Elgar Publishing, pp. 128-46.

Grunow D. and M. Evertsson (eds) (2016), Couples' Transitions to Parenthood: Analysing Gender and Work in Europe, Cheltenham, UK and Northampton, MA, USA: Edward Elgar Publishing.

Hareven, T.K. and K. Masaoka (1988), 'Turning points and transitions: Perceptions of the life course', Journal of Family History, 13 (3), 271-89.

Heaton, J. (2008), 'Secondary analysis of qualitative data', in P. Alasuutari, L. Bickman, and J. Brannen (eds), The Sage Handbook of Social Research Methods, London: Sage, pp. 506-19.

Heinz, W.R. (2016), 'Conceptual foundations of qualitative life course research', Sociologia, Revista da Faculdade de Letras da Universidade do Porto (Special issue: Famílias e Curso de Vida. Potencialidades, limites e desafios metodológicos), 20-37. doi:10.21747/doi.org/0872-3419/tema3.

Heinz, W.R., U. Kelle, A. Witzel, and J. Zinn (1998), 'Vocational training and career development in Germany: Results from a longitudinal study', International Journal for Behavioral Development, 22, 77-101. https://doi.org/10.1080/016502598384522.

Hermanowicz, J. (2003), 'Longitudinal qualitative research', in M. Shanahan, J. Mortimer, and M. Johnson (eds), Handbook of the Life Course, New York: Springer, pp. 491-513.

Holland J., R. Thomson, and S. Henderson (2006), 'Qualitative longitudinal research', Discussion paper, Families and Social Capital, ESRC Research Group Working Paper 21.

Hollstein, B. (2002), Soziale Netzwerke nach der Verwitwung: Eine Rekonstruktion der Veränderungen informeller Beziehungen, Opladen: Leske + Budrich.

Hughes, K. and A. Tarrant (eds) (2020), Qualitative Secondary Analysis, London: Sage.

Irwin, S. and M. Winterton (2014), 'Gender and work-family conflict: A secondary analysis of "Timescapes" data', in J. Holland and R. Edwards (eds), Understanding Families over Time: Research and Policy, London: Palgrave Macmillan, 142-60.

Irwin, S., J. Bornat, and M. Winterton (2012), 'Timescapes secondary analysis: Comparison, context and working across datasets', Qualitative Research, 12 (1), 66-80. doi:10.1177/1468794111426234. 
Johnson, M.K. (2002), 'Social origins, adolescent experiences, and work value trajectories during the transition to adulthood', Social Forces, 80, 1307-41.

Keddi, B., P. Pfeil, P. Strehmel, and S. Wittmann (2003), 'Beyond typical life-plans: Young women's themes of life in East and West Germany', in E. Kolinsky and H.M. Nickel (eds), Reinventing Gender, London/Portland: Frank Cass Publishers, pp. 190-208.

Koro-Ljungberg, M. and R. Bussing (2013), 'Methodological modifications in a longitudinal qualitative research design', Field Methods, 25 (4), 423-40. doi:10.1177/1525822X12472877.

Larenza, O. (2019), Social Policy Shaping the Life-Course: A Study on Lone Parents' Vulnerability, Doctoral dissertation, University of Lausanne.

Legewie, N. and I. Tucci (2020), 'Studying turning points in labour market trajectories: Benefits of a panel-based mixed methods design', Longitudinal and Life Course Studies.

Longo, M.L., S. Bourdon, J. Charbonneau, C. Kornig, and V. Mora (2013), 'Normes sociales et imprévisibilités biographiques. Une comparaison, France, Québec et Argentine', Agora-Débats Jeunesses, 65, 93-108.

Mazzucato, V. (2015), 'Transnational families and the well-being of children and caregivers who stay in origin countries', Social Science and Medicine, 132, 208-14. doi:10.1016/j.socscimed.2014.11.030.

McAdams, D.P. (2005), 'Studying lives in time: A narrative approach', Advances in Life Course Research, $10237-58$.

McAdams, D.P. (2008), 'Personal narratives and the life story', in O.P. John, R.W. Robins, and L.A. Pervin (eds), Handbook of Personality: Theory and Research, New York: Wilford Press, pp. 242-62.

McKie, L., S. Bowlby, and S. Gregory (2001), 'Gender, caring and employment in Britain', Journal of Social Policy, 30 (2), 233-58. doi:10.1017/S0047279401006262.

McKie, L., S. Gregory, and S. Bowlby (2002), 'Shadow times: The temporal and spatial frameworks and experiences of caring and working', Sociology, 36 (4), 897-924. doi:10.1177/003803850203600406.

Millar, J. and T. Ridge (2013), 'Lone mothers and paid work: The "family-work project", International Review of Sociology: Revue Internationale de Sociologie, 23 (3), 564-77. doi:10.1080/03906701 .2013 .856161 .

Neale, B. (2020), What Is Qualitative Longitudinal Research, London: Sage.

Neale, B. and J. Flowerdew (2003), 'Time, texture and childhood: The contours of longitudinal qualitative research', International Journal of Social Research Methodology, 6 (3), 189-99. doi:10.1080/ 1364557032000091798.

Neale, B. and R. Patrick (2016), 'Engaged young fathers? Gender parenthood and the dynamics of relationships', Following Young Fathers Working Paper Series, 1.

Pearson, A. (2012), 'Seven Up! A tale of two Englands that, shamefully, still exist', The Daily Telegraph, accessed 3 July 2020 at www.telegraph.co.uk/comment/columnists/allison-pearson/9269805/Seven -Up-A-tale-of-two-Englands-that-shamefully-still-exist.html.

Pollard, A. (2007), 'The identity and learning programme: "Principled pragmatism" in a 12-year longitudinal ethnography', Ethnography and Education, 2 (1), 1-19.

Riessman, C.K. (1991), 'Beyond reductionism: Narrative genres in divorce accounts', Journal of Narrative and Life History, 1, 41-68.

Rousseau, C. (2018), 'Les bonnes conditions: Chronique de jeunes presque sans histoires', Le Monde, accessed 3 July 2020 at www.lemonde.fr/televisions-radio/article/2018/05/17/les-bonnes-conditions -chronique-de-jeunes-presque-sans-histoires 5300150 1655027.html.

Ryan, L., M. Lopez Rodriguez, and P. Trevena (2016), 'Opportunities and challenges of unplanned follow-up interviews: Experiences with Polish migrants in London', Forum: Qualitative Social Research, 17 (2). www.qualitative-research.net/index.php/fqs/article/view/2530/3988.

Saldana, J. (2003), Longitudinal Qualitative Research, Analyzing Change through Time, New York: Altamira Press.

Salmons, J. (2015), Qualitative Online Interviews, Thousand Oaks, CA: Sage.

Sanchez Mira, N. and L. Bernardi (2020), 'Relative time in life course research', Longitudinal and Life Course Studies. doi:10.1332/175795920X15918713165305.

Scott, J. and D. Alwin (1998), 'Retrospective versus prospective measurement of life histories in longitudinal research', in J.Z. Giele and G.J. Elder, Jr. (eds), Methods of Life Course Research: Qualitative and Quantitative Approaches, Thousand Oaks, CA: Sage, pp. 98-127. doi:10.4135/9781483348919. 


\section{Research handbook on the sociology of the family}

Shirani F., K. Henwood, and C. Coltart (2012), 'Meeting the challenges of intensive parenting culture: Gender, risk management and the moral parent', Sociology, 46 (1), 25-40. doi:10.1177/ 0038038511416169 .

Singer, J.A. (2004), 'Narrative identity and meaning making across the adult lifespan: An introduction', Journal of Personality, 72 (3), 437-59. doi:10.1111/j.0022-3506.2004.00268.x.

Smart, C. and B. Neale (2007), Family Fragments, Cambridge: Polity Press.

Smart, C., B. Neale, and A. Wade (2001), The Changing Experience of Childhood: Families and Divorce, Oxford: Polity Press.

SmithBattle, L., R. Lorenz, C. Reangsing, J.L. Palmer, and G. Pitroff (2018), 'A methodological review of qualitative longitudinal research in nursing', Nursing Inquiry, 25 (4), e12248. doi:10.1111/nin .12248 .

Struffolino E., L. Bernardi, and O. Larenza (2020), 'Lone parenthood and employment trajectories: A longitudinal mixed-method study', Comparative Population Studies, 45, 265-98. doi:10.12765/ CPoS-2020-14.

Thomson R. and J. McLeod (2015), 'New frontiers in qualitative longitudinal research: An agenda for research', International Journal of Social Research Methodology, 18 (3), 243-50. doi:10.1080/ 13645579.2015.1017900.

Thomson, R., L. Plumridge, and J. Holland (2003), 'Editorial: Longitudinal qualitative research: A developing methodology', International Journal of Social Research Methodology, 6 (3), 185-7. doi: 10.1080/1364557032000091789.

Thomson, R., L. Berriman, and S. Bragg (2018), Researching Everyday Childhoods: Time, Technology and Documentation in a Digital Age, London: Bloomsbury Academics.

Thurman, S.L. (2015), 'Ethical considerations in longitudinal studies of human infants', Infant Behavior and Development, 38, 116-25. doi:10.1016/j.infbeh.2014.12.012.

Vogl, S. and U. Zartler (2021), 'Interviewing adolescents through time: Balancing continuity and flexibility in a qualitative longitudinal study', Longitudinal and Life Course Studies, 12, 1, 83-97. https:// doi.org/10.1332/175795920X15986464938219.

Yates, L. and J. McLeod (1996), 'And how would you describe yourself? Researchers and researched in the first stages of a qualitative, longitudinal research project', Australian Journal of Education, 40 (1), 88-103. doi:10.1177/000494419604000106.

Weller, S. (2010), 'Time(s) to be creative! Sustaining young people's engagement in qualitative longitudinal research', in F. Shirani and S. Weller (eds), Conducting Qualitative Longitudinal Research: Fieldwork Experiences, Leeds: University of Leeds, pp. 34-48.

Woodman, D. (2011), 'Young people and the future: Multiple temporal orientations shaped in interaction with significant others', YOUNG, 19 (2), 111-28. doi:10.1177/110330881001900201.

Wyatt, D. (2016), 'Born to be different, Channel 4 - TV review: A life-affirming watch', The Independent, accessed July 2020 at www.independent.co.uk/arts-entertainment/tv/reviews/born-to-be -different-channel-4-tv-review-a-truly-life-affirming-watch-a6905526.html. 